\title{
False periods in complex chaotic systems
}

\author{
V. Votruba ${ }^{1,2}$, P. Koubský ${ }^{1}$, D. Korčáková ${ }^{1}$, and F. Hroch ${ }^{2}$ \\ 1 Astronomický ústav, Akademie věd České republiky v.v.i., 25165 Ondřejov, Czech Republic \\ e-mail: votruba@sunstel.asu.cas.cz \\ 2 Ústav teoretické fyziky a astrofyziky, Přirodověcká fakulta, Masarykova univerzita, Kotlářská 2, 61137 Brno, Czech Republic \\ Received 16 October 2008 / Accepted 19 December 2008
}

\section{ABSTRACT}

\begin{abstract}
Aims. Astrophysical objects frequently exhibit some irregularities or complex behaviour in their light curves. We focus primarily on hot stars, where both radial and non-radial pulsations are observed. One of the primary research goals is to determine physical parameters of stellar pulsations by analyzing their light curves or spectra, focusing on periodic or quasiperiodic behaviour.

Methods. We analyse the feasibility of classical methods for period searches in a nonlinear chaotic system, such as the Rössler system, where a period does not exist at all. As an astrophysical application of the chaotic system, we utilize a simple model of stellar pulsation with two different sets of parameters corresponding to periodic and chaotic behaviour. For both models we create a synthetic signal, and then apply widely used methods for period finding, such as the phase dispersion method and periodograms. For comparison, a quasi-periodic signal is employed as well.

Results. The period analysis indicates periods even for the chaotic signal. Such periods are apparently spurious. This implies that it is very problematic to distinguish chaotic and quasiperiodic process by such an analysis only.
\end{abstract}

Key words. stars: oscillations - stars: variables: Cepheids - chaos

\section{Introduction}

Variability of astronomical sources like stars is an important aspect of their observation. We can detect variability in their light and/or spectra on different timescales. Dynamical pulsations, radial or non-radial, may be one of the main sources of intrinsic variability in many types of stars. As was shown by Eddington (1919), stars may exhibit pulsation due to the unstable layers in the stellar envelope. The first theoretical modelling of pulsations predicted regular oscillations and such modelling was succesfully applied to objects like classical Cepheids. But later, observations indicated other types of pulsating stars, e.g. Population II Cepheids, which in some cases exhibit irregular patterns in observational data.

There was no reliable explanation of such variability until the pioneering work of Baker et al. (1966), which showed that a simple one-zone model of stellar oscillators with a nonlinear adiabatic term can in some specific conditions produce irregular pulsation. Similar results were also obtained by Moore \& Spiegel (1966), Auvergne \& Baglin (1985) and Auvergne (1988). The disadvantage of these models is their dynamically unstable equilibrium state, which seems to be nonrealistic for stars. Takeuti (1987) and later Saitou \& Takeuti (1989) showed that similar, yet dynamically stable and more precise one-zone model also produced chaotic solutions, with a strange attractor similar to the Rössler one (see Rössler 1976). Detailed, more realistic calculations of the state equation for helium ionization in a one zone model, which was performed by Buchler \& Regev (1982), confirmed that pulsation instability may lead to chaotic behaviour.

Goupil et al. (1986) studied light curves of the pulsating white dwarf PG 1351+489 and found strong evidence of period doubling. Period doubling is a scenario where a system that primarily oscillates with a fundamental frequency $v_{0}$, after change of the control parameter oscillates with frequency $v_{0} / 2$ (see Helleman 1980; Gurel \& Rössler 1979). Cascades of period doubling is a typical transition routes to chaotic behaviour. Such an effect is typical of chaotic systems. Serre et al. (1996) analysed long time-series of photometric observations of the variable star R Scuti, one possible candidate pulsating star which exhibits a strange irregular light curve. By using the method of global flow reconstruction (see more about global time series predictions in Brown 1993; Abarbanel et al. 1994), they were able to reproduce the observation with the chaotic dynamics very well. More details and examples of chaotic pulsations can be found in Buchler (1987).

These clearly show that there is both theoretical and experimental evidence of quasiperiodic or chaotic behaviour in variable stars. The problem is how to distinguish between these two possibilities, if only limited time series are available, often very unevenly sampled. In other words, what will be the result, if classical astronomical tools for the period searches, such as periodograms, the phase dispersion minimalization method or more sophisticated techniques are applied to data originating from quasiperiodic or chaotic sources. In the next paragraphs we tackle the problem using synthetic data.

\section{Generation of synthetic time series}

As an example of irregular stellar variability due to pulsation we use the model of Tanaka \& Takeuti (1988). In this model, the set of ordinary differential equations (ODE) for a stellar oscillator with nonlinear, nonadiabatic terms, reads

$$
\begin{aligned}
& \frac{\mathrm{d} x}{\mathrm{~d} t}=y \\
& \frac{\mathrm{d} y}{\mathrm{~d} t}=\alpha+\mu y+z \\
& \frac{\mathrm{d} z}{\mathrm{~d} t}=-\beta y-p z-q y+s y z
\end{aligned}
$$



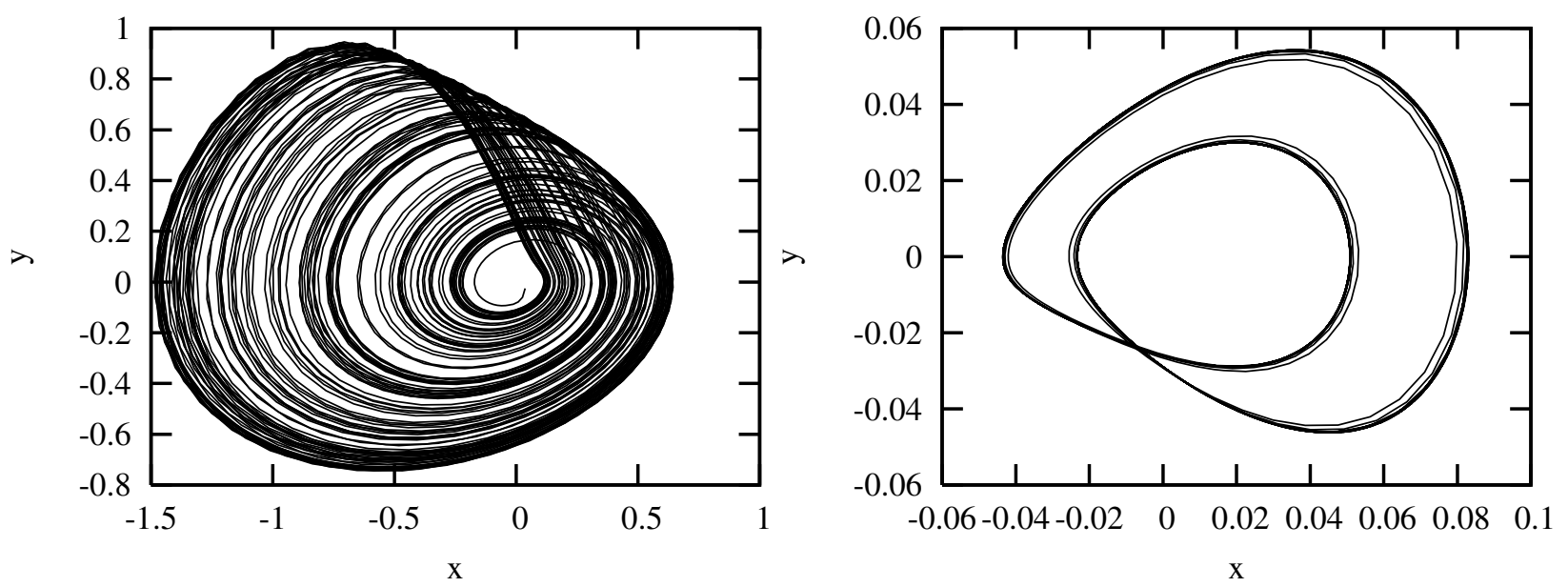

Fig. 1. Left panel: chaotic attractor constructed from a numerical solution of the ODE system (1) with the parameters from Table 1. - chaotic case. Right panel: two-limit cycle attractor constructed from numerical solution of (1) with the parameters from Table 1. - periodic case.

Table 1. Parameters of the stellar pulsation model.

\begin{tabular}{ccccccccc}
\hline \hline & $\alpha$ & $\beta$ & $\mu$ & $p$ & $q$ & $s$ & $\sigma$ & $\bar{x}$ \\
\hline periodic & -0.5 & 0.5 & 0.5 & 3.2 & 0.5 & 1.0 & 0.2 & 0 \\
chaotic & -0.5 & 0.5 & 0.5 & 4.0 & 0.5 & 1.0 & 0.3 & 0 \\
\hline
\end{tabular}

Table 2. Parameters for the quasiperiodic signal.

\begin{tabular}{cccccccc}
\hline \hline$a_{1}$ & $a_{2}$ & $a_{3}$ & $f_{1}$ & $f_{2}$ & $f_{3}$ & $\sigma$ & $\bar{x}$ \\
\hline 0.4 & 0.6 & 0.5 & $\sqrt{5}$ & $\sqrt{3}$ & $\sqrt{2}$ & 0.1 & 0 \\
\hline
\end{tabular}

where the first two equations represent equations of motion and the last one is a state equation with a nonadiabatic term. We can adjust the behaviour of the model using different values of $\alpha, \beta, \mu, p, q, s$. The system of ODE of the first order was numerically solved with the Runge-Kutta method for two different sets of model parameters, the first one corresponding to the periodic regime with a two-limit cycle attractor and the second one to the aperiodic, chaotic regime with a strange attractor similar to the Rössler type. The parameters are summarized in Table 1.

We use the initial conditions $\left(x_{0}=0.37, y=-0.249, z=\right.$ -0.174 ) for the chaotic solution. The initial conditions are very close to the orbit of the attractor in phase space. We obtain a time-dependence of the state variables $x(t), y(t), z(t)$ in the form of a time series with an equidistant time step. We choose one state variable, namely $x(t)$, as a representation of the measured variable. To simulate a synthetic signal from the quasiperiodic process, a different approach is employed. The signal is modeled by the following equation

$x_{\text {quasi }}(t)=a_{1} \sin \left(f_{1} t\right)+a_{2} \sin \left(f_{2} t\right)+a_{3} \sin \left(f_{3} t\right)$

where $a_{1}, a_{2}, a_{3}$ are amplitudes of incommensurate modes with frequencies $f_{1}, f_{2}, f_{3}$. Their values are summarized in Tables 1 and 2 . These parameters are carefully chosen in order to obtain similar dependencies of $x(t)$ and $x_{\text {quasi }}(t)$ for the chaotic and quasiperiodic case, namely amplitudes of oscillations (see Fig. 2). The computed signal for each type of process is plotted in Fig. 2. Since we want to simulate real measurements, we add a synthetic signal with Gaussian noise. It is important that for a simulation of light curves from objects others than stars, such as QSOs, noise may by colored and then it is necessary to use a different type of noise, e.g. Poisson noise. The noise can be described using a normal distribution

$G(x)=\frac{1}{\sigma \sqrt{2 \pi}} \exp \left((x-\bar{x})^{2} / 2 \sigma^{2}\right)$

where $\sigma$ is the variance and $\bar{x}$ is the mean value of the measurement errors. For numerical simulations of the noise with a normal distribution, we use the method of Hamming (1962). It was necessary to set the different values of $\sigma$ for each type of signal in order to have similar $\mathrm{S} / \mathrm{N}$ ratios.

Data for synthetic time series satisfy the condition $t>300$, i.e. reasonably far away from the starting point $(t=0)$. It is necessary to make sure that the solution is relaxed away from the influence of the initial conditions.

It can be readily seen that chaotic and quasiperiodic oscillations (see Fig. 2) have very similar patterns, different to the simple periodic signal (two-limit cycle).

\section{Analysis of the signal}

\subsection{Classical period analysis}

The most common techniques used for period searches are the power spectral analysis (PWS), the modified Lomb periodogram (LPD), the phase dispersion minimalization (PDM), and the string length method (SLM). In the first case, discrete Fourier transformation is applied to a dataset resulting in a power spectrum (for more details see e.g. Scargle 1982). For unevenly sampled series it is better to use a modification of PWS, method LPD (see for more details Lomb 1976). The third method binds data in a grid of phases and minimizes phase dispersion statistics (see for more details Stellingwerf 1978; Lafler\& Kinman 1965). The SLM method is similar to the previous one (see Dworetsky 1983), and the LPD method behaves like the PWS. So we limit on the two methods, PWS and PDM only. Obviously, PDM and PWS techniques applied on a periodic signal detect corresponding frequencies without any doubt, but, as we will show, a different situation arises for quasiperiodic and chaotic regimes.

1. Quasiperiodic signal. The PWS analysis of the quasiperiodic signal produced by known incommensurate frequencies (see Table 2) detects the frequencies used. Moreover, it is possible to recognize the leading frequency, which is in our case $f_{2}=\sqrt{3}$. The noise in the periodogram in Fig. 4 is 


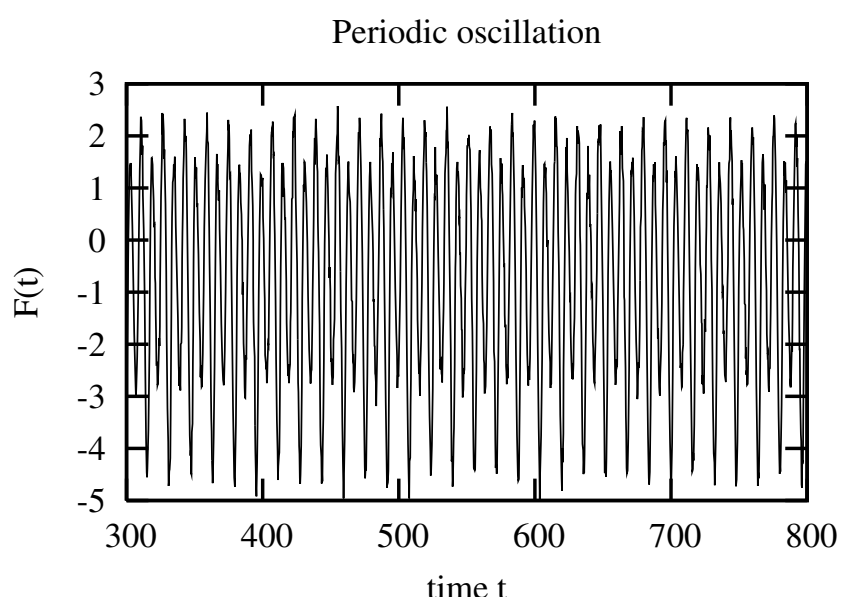

Chaotic oscillation

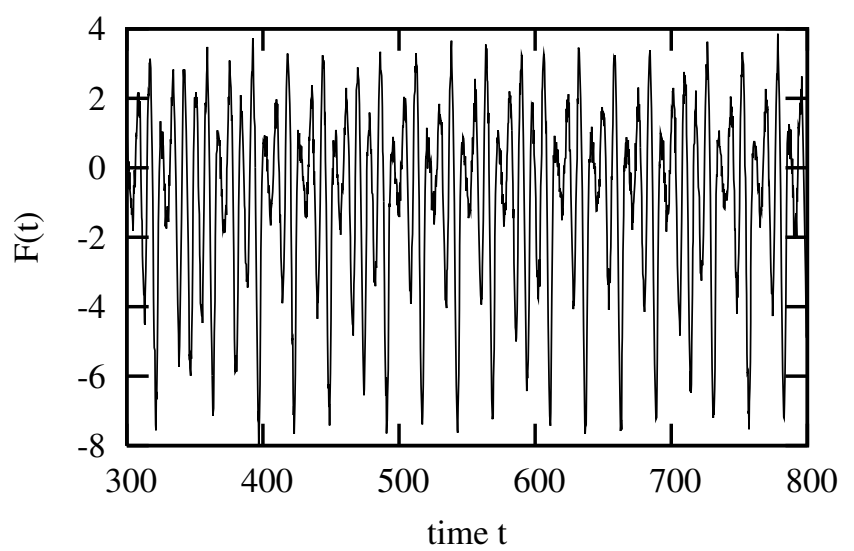

Quasiperiodic oscillation

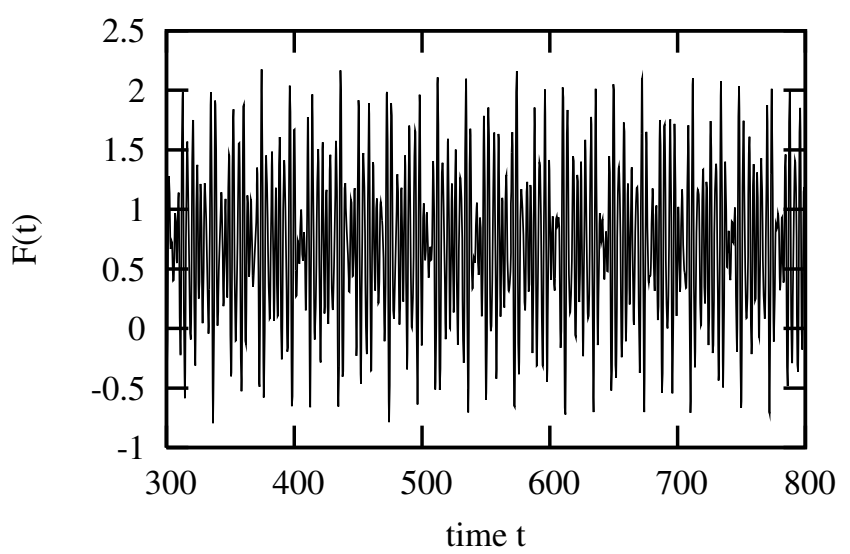

Gaussian noise

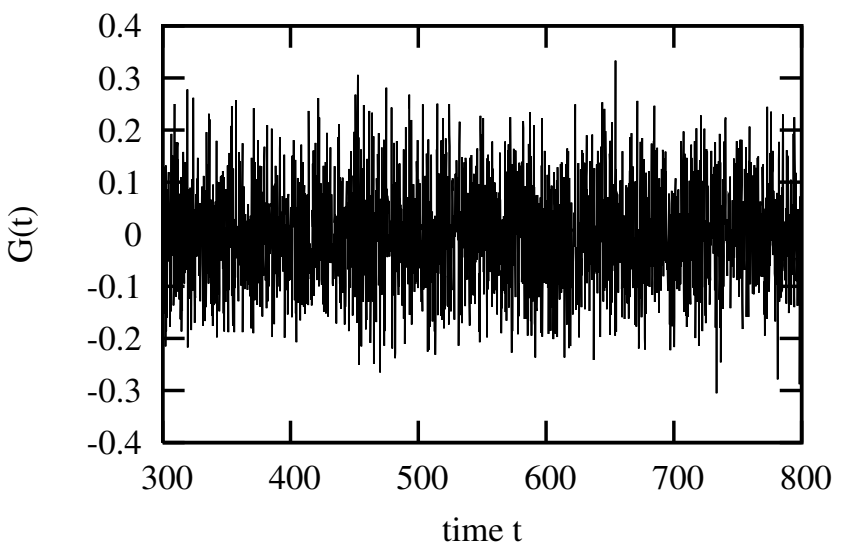

Fig. 2. Upper left panel: $x(t)$ solution of the ODE system (1) for the periodic case with a two-limit cycle. Upper right panel: $x(t)$ solution of Eq. (2) for the quasiperiodic case. Lower left panel: $x(t)$ solution of the ODE system (1) for the chaotic case. Lower right panel: Gaussian noise (3) with parameters $\sigma=0.1$ and $\bar{x}=0$. All physical quantities are in arbitrary units.
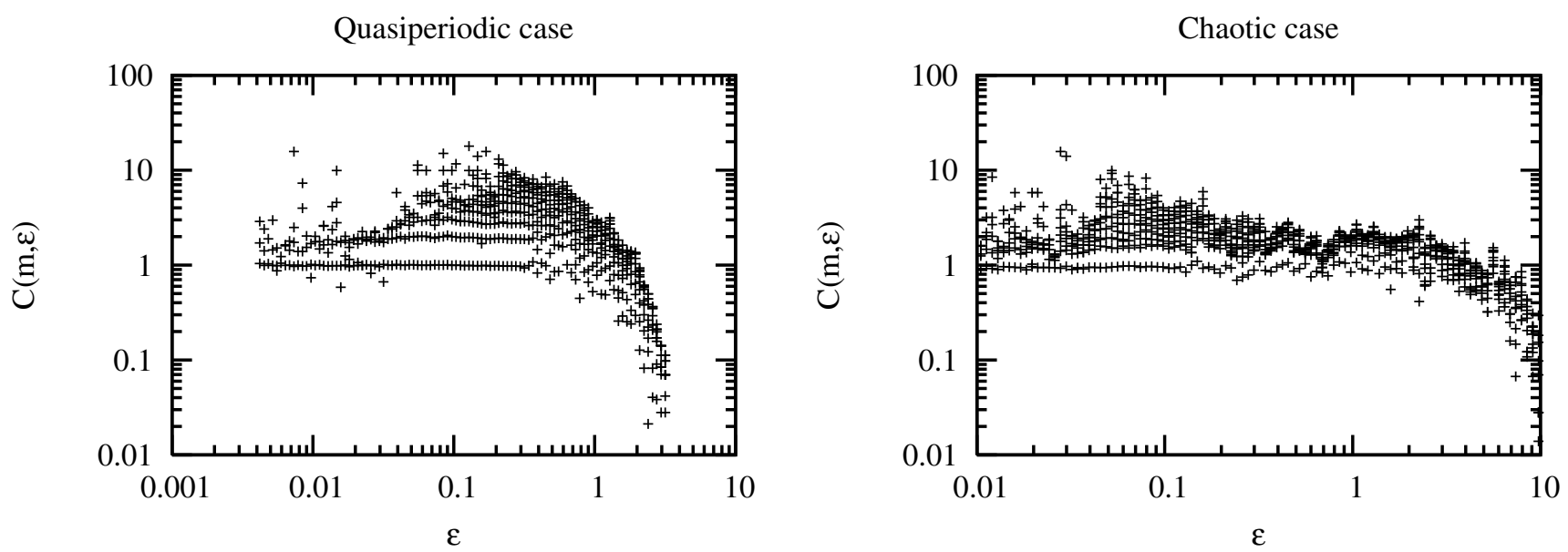

Fig. 3. Correlation integrals (4) for different lengthscales $\epsilon$ and embedding dimensions $m$. The quasiperiodic case is in the left panel, chaotic case is in the right panel.

caused by the simulated Gaussian noise of the signal. The PDM method yields a similar estimate of the input frequencies, and the results also show some other frequencies that are subharmonics or harmonics of the frequency set. The phase diagram in Fig. 5 (on the left) is folded with the leading frequency $f_{2}$. Clearly, the structure is not a simple sine wave. It is a quasiperiodic, and hence it is composed of a set of harmonics with slightly different phase. After accounting for Gaussian noise, this difference is smeared out.

2. Chaotic signal. The results become much more interesting for the chaotic signal. Again, both PWS and PDM methods detect periods, as can be clearly seen in Fig. 4. After some checks we can recognize the two longest independent periods. The power spectrum of the chaotic signal shows the most 
Power spectrum (q)
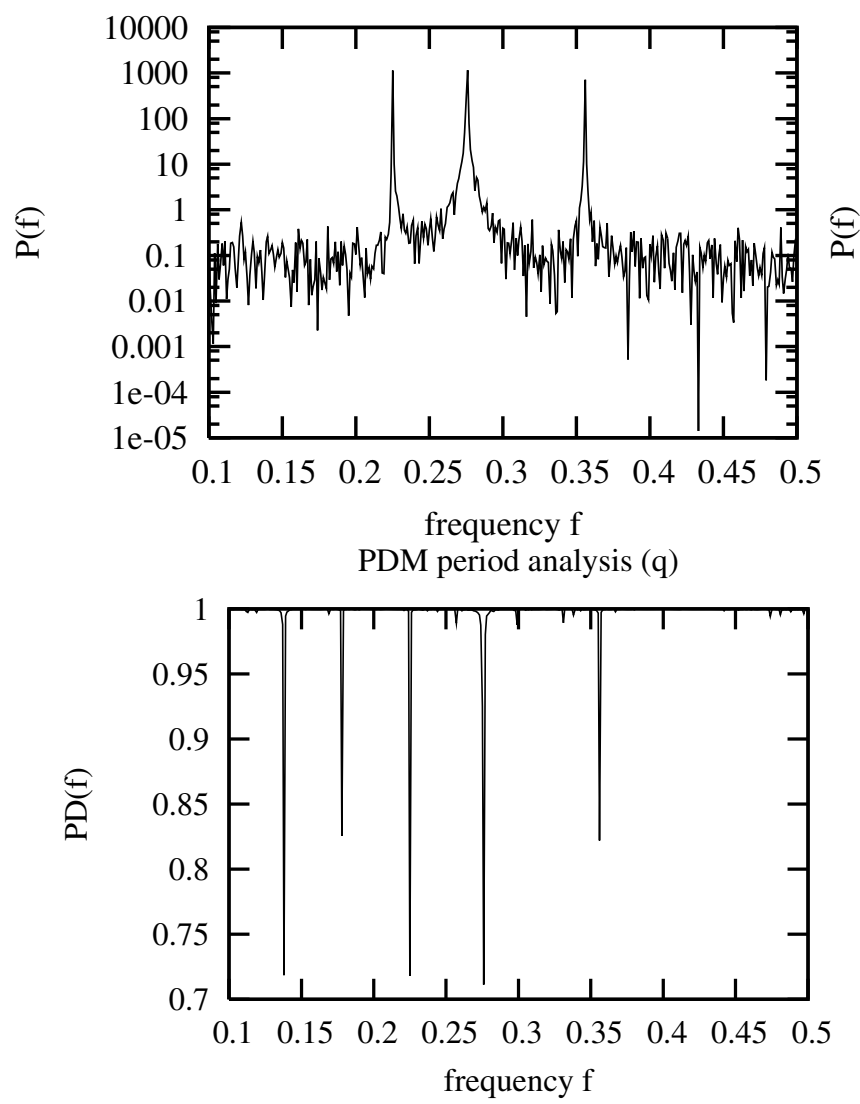

Power Spectrum (ch)

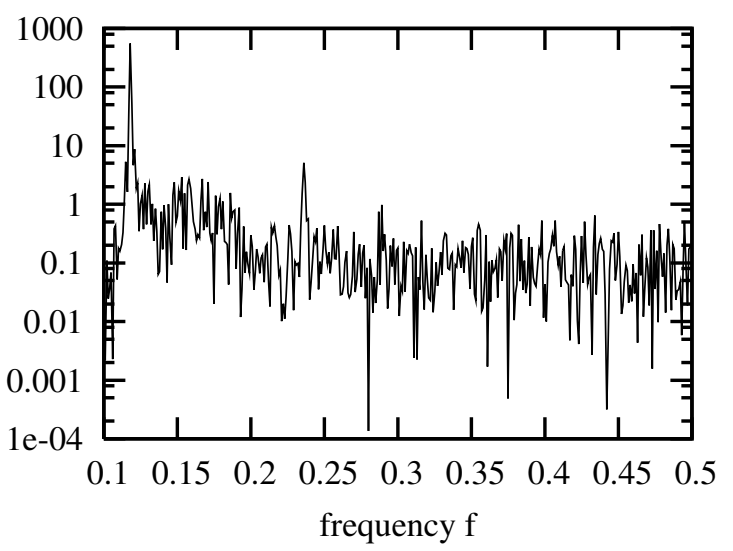

PDM period analysis (ch)

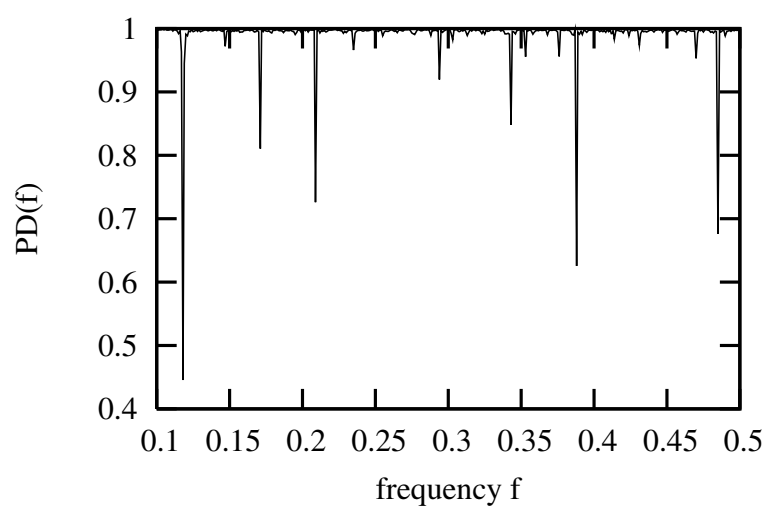

Fig. 4. Upper panels: power spectra constructed for quasiperiodic oscillation (on the left) described by (2) and the chaotic case of stellar oscillation (on the right) described by (1). Bottom panels: periodogram constructed using the phase dispersion minimalization method for the quasiperiodic case (on the left) and chaotic case (on the right). For the frequency we used arbitrary units.

significant frequencies together with the frequency noise. Nevertheless, low-level noise is present even if Gaussian noise is not applied. It is the consequence of the chaotic behaviour.

Theoretically, power spectra of the chaotic data are composed of an infinite number of frequencies. This is true, however, only if there is an infinite number of observations (see Regev 2006, Chap. 5). In reality, there is only a limited number of observations, which are, furthermore, unevenly spaced. The phase diagram constructed for the most significant frequency derived $f_{1}=0.1179$ is shown in Fig. 5 (on the right). In the phase diagram without Gaussian noise, we can recognize a two-wave structure in the phase plot. On the other hand, when Gaussian noise is applied a simple noisy sine wave can be identified. This is similar to the quasiperiodic case results, although the data originated from a nonlinear chaotic process, where period has no meaning. Other frequencies $f_{2}, f_{3}$ lead to even less clear phase diagrams.

The above results clearly show that it is very difficult to distinguish between chaotic and quasiperiodic cases using period analysis only. Thus, we must look for other methods, which can help to resolve both processes.

\subsection{Nonlinear analysis}

For detailed analysis of the signal that can be generated by nonlinear chaotic processes, we can use methods of nonlinear time series analysis. These methods were developed for classification and identification of chaotic phenomenon in time series of the experimental data (see review Abarbanel et al. 1993). As a indication of chaotic behaviour we can use topological invariants, namely the correlation dimension and Lyapunov exponents. Algorithms for numerical estimates of such invariants from time series are described by Abarbanel et al. (1993) and by Kantz \& Schreiber (2004). In our case the indicator of chaotic behaviour is the correlation integral defined (see more detail Kantz \& Schreiber 2004) as

$C(m, \epsilon)=\frac{2}{\left(N-n_{\min }\right)\left(N-n_{\min }-1\right)} \sum_{i=1}^{N} \sum_{j=i+1+n_{\min }}^{N} \theta\left(\epsilon-\left\|\boldsymbol{s}_{i}-\boldsymbol{s}_{j}\right\|\right)(4)$

where $m$ is the embedding dimension, $\boldsymbol{s}_{n}$ denotes the delay embedding vector, $\epsilon$ is the lengthscale parameter, and $N$ is the number of points. A possible problem with temporal correlations is overcome by excluding pairs that are too close in time (parameter $n_{\min }$ ). Before we apply the procedure for calculation of the correlation dimension to the noisy signal (chaotic or quasiperiodic), it is necessary to clean the noisy data. This is done by using nonlinear noise reduction with locally constant approximations - the simple nonlinear filter described by Schreiber (1993). The results of the calculation are shown in Fig. 3. The chaotic processes are characterized by a plateau in the plot of $C(m, \epsilon)$ for different embedding dimensions (left panel Fig. 3), which indicates a self-similar geometry. A linear 


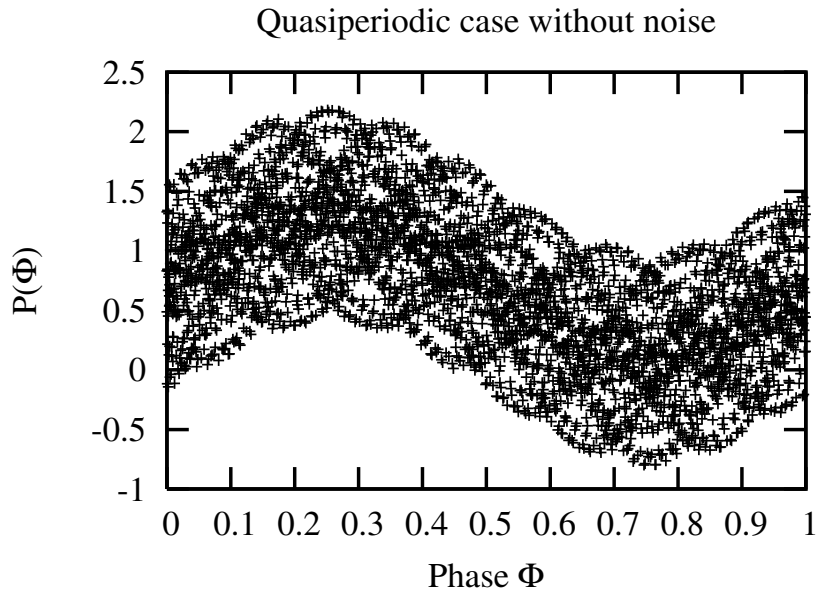

Quasiperiodic case with noise

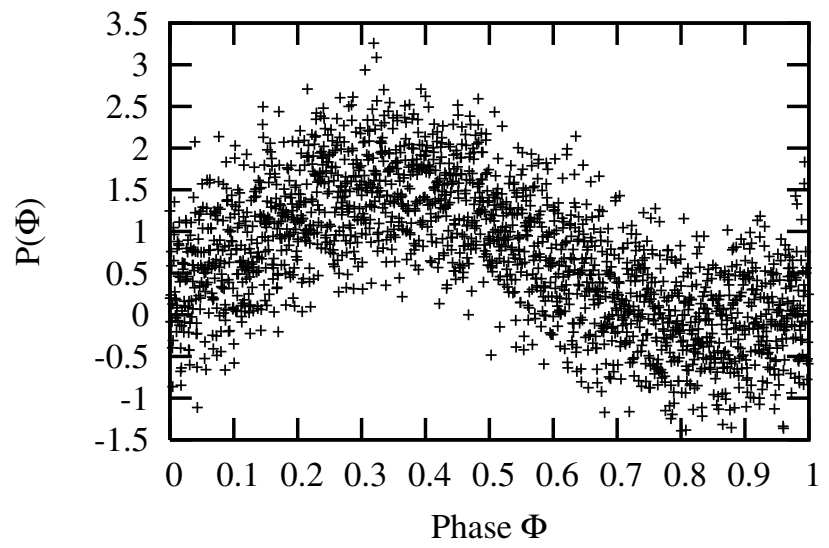

Chaotic case without noise

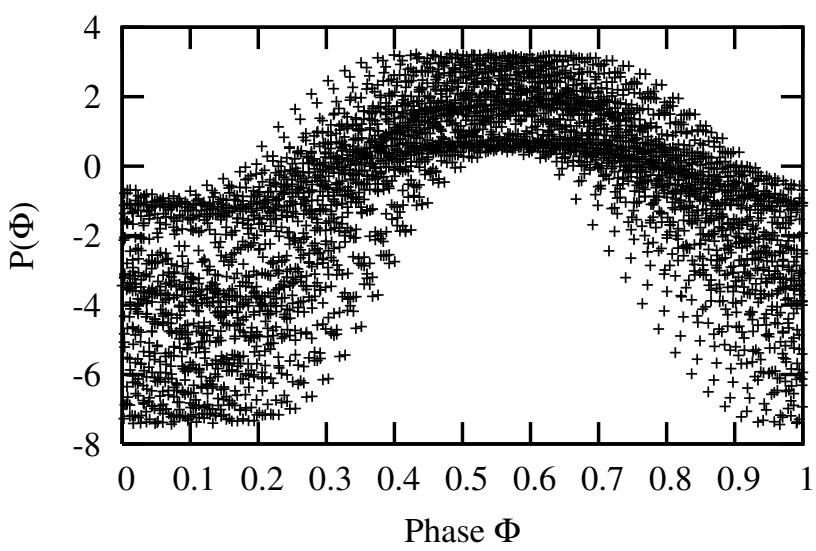

Chaotic case with noise

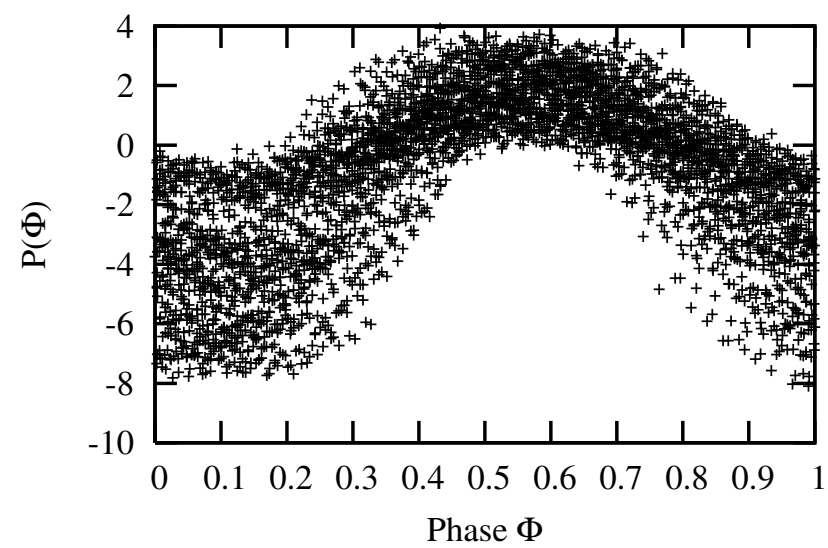

Fig. 5. Left upper panel: phase diagram for the quasiperiodic case and the dominant frequency $f_{1}=\sqrt{3}$ without noise. Right upper panel: final phase diagram for the frequency $f_{1}=0.1176$ of the chaotic case. Left bottom panel: the same as the left upper panel but with Gaussian noise. Right bottom panel: the same as the right upper panel, but with Gaussian noise. On the bottom panels both diagrams are similar, showing a simple sine-wave pattern. All physical quantities are in arbitrary units.

fit to this plateau region leads to the estimation of correlation dimension

$C_{\text {dim }}=1.90 \pm 0.08$.

This value agrees with the typical value of the Rössler type attractor. In the case of the quasiperiodic signal we cannot identify the plateau region (right panel Fig. 3). This indicates that there is no self-similar geometry for this type of the signal.

\section{Summary}

Analysis of synthetic signals simulating quasiperiodic and chaotic data led to the following findings:

1) In the realistic case (finite number of observations with Gaussian noise), it is impossible to distinguish between quasiperiodic and chaotic behaviour by classical methods of period analysis.

2) When the data contain large amounts of noise, the chaotic or quasiperiodic character of the signal can be overlooked and a simple periodic solution with the strongest frequency can be determined from the period analysis.

3) In order to definitely rule out presence of chaos it is neccessary to use a nonlinear time series analysis. Thus, we must reconstruct the topologically similar phase portrait of the system using the technique described by Abarbanel et al. (1993). Then we must determine the invariants, such as the correlation dimension or Lyapunov coefficients from the reconstructed time series, and from their values we can decide if deterministic chaos is present in the data or not. However, both these procedures need better quality of data (noise, length of time series).

4) We would like to draw attention to the fact that irregular chaotic behaviour may be more usual in astronomical data than thought before, and that some systems that were identified as quasiperiodic may actually be chaotic ones.

Acknowledgements. We thank S. Shore and T. Dytrych for helpful comments on our analysis. This research has made use of NASA's Astrophysics Data System. This work was supported by the grant CZ 205/09/P476. The Astronomical Institute Ondřejov is supported by project AV0 Z10030501.

\section{References}

Abarbanel, H. D. I., Brown, R., Sidorowich, J. J., \& Tsimring, L. S. 1993, Rev. Mod. Phys., 65, 1331

Abarbanel, H. D. I., Carroll, T. A., Pecora, L. M., Sidorowich, J. J., \& Tsimring, L. S. 1994, Phys. Rev. E, 49, 1840

Auvergne, M., \& Baglin, A. 1985, A\&A, 142, 388

Auvergne, M. 1988, A\&A, 204, 341

Baker, N. H., Moore, D. W., \& Spiegel, E. A. 1966, ApJ, 71, 844 
Brown, R. 1993, Phys. Rev. E, 47, 3962

Buchler, J. R., \& Regev, O. 1982, ApJ, 263, 312

Buchler, J. R. 1987, Chaotic Phenomena in Astrophysics, ed J. R. Buchler, \& H. Eichhorn, Annals New York Academy of Sciences, 37

Dworetsky, M. M. 1983, MNRAS, 203, 917

Eddington, A. S. 1919, MNRAS, 79, 2

Goupil, M. J., Auvergne, A., \& Baglin, A. 1986, A\&A, 196, L13

Gurel, O., \& Rössler, O. E. 1979, Bifurcation Theory and Applications to Scientific Disciplines, Annals New York Academy of Science, 316

Hamming, R. W. 1962, Numerical methods fo Scientist and Engineers, (McGraw-Hill)

Helleman, R. ed. 1980, Nonlinear dynamics, Annals New York Academy of Science, 357

Kantz, H., \& Schreiber, T. 2004, Nonlinear Time Series Analysis (Cambridge University Press)
Lafler, J., \& Kinman, T. D. 1965, ApJS, 11, 216

Lomb, N. R. 1976, Ap\&SS, 39, 447

Moore, D. W., \& Spiegel, E. A. 1966, ApJ, 143, 871

Omodei, N., Bellazzini, J., \& Montangero, S. 2004, A\&A, 414, 1177

Regev, O. 2006, Chaos and Complexity in Astrophysics (Cambridge University Press)

Rössler, O. E. 1976, Phys. Rev. Lett., 57, 397

Saitou, M., \& Takeuti, M. 1989, PASJ, 41, 297

Scargle, J. D. 1982, ApJ, 263, 835

Serre, T., Kolláth, Z., \& Buchler, J. R. 1996, A\&A, 311, 833

Schreiber, T. 1993, Phys. Rev. E, 47, 2401

Stellingwerf, R. F. 1978, ApJ, 224, 953

Takeuti, M. 1987, Ap\&SS, 136, 129

Tanaka, Y., \& Takeuti, M. 1988, Ap\&SS, 148, 229 